Die Veröffentlichung des "Corpus Confessionum erfolgt in Lieferungen von je 8o Seiten im Lexikon-Oktav-Format; in jedem Vierteljahr werden zwei Lieferungen erscheinen. Das Werk wird somit in jedem Jahr um mehr als 600 Seiten wachsen. Die Lieferungen werden zu Bänden zusammengefaßt. In Aussicht genommen sind etwa zwanzig Bände, die im Laufe von ungefähr Io Jahren erscheinen sollen. Der Bezugspreis beträgt bei Subskription auf das ganze Werk in der Regel M. 7.- für jede Lieferung von 5 Bogen $=80$ Seiten. Lieferungen kleineren oder größeren Umfanges, die sich am Schḷß der einzelnen Bände ergeben können, werden einen entsprechend niedrigeren oder höheren Preis haben.

\title{
PLAN DES WERKES
}

I. Ökumenische Grundlagen.

2. Orthodox-katholische Kirche des Ostens.

3. Schismatische Kirchen d.Ostens.

4. Russische Sondergruppen.

5. Römisch-katholische Kirche.

6. Altkatholizismus und Modernismus.

7. Deutsches Luthertum.

8. Deutsch-reformiertes Christentum.

9. Deutsch-evangelische Landesund Freikirchen.

10. Deutsche Erweckungs- und Heiligungsbewegungen.

II. Deutsche Aufklärung, theologische Richtungen und philosophisch-humanitäre Bewegungen.

12. Evangel. Kirchen der Schweiz.
I 3. Evangel. Kirchen der Nieder. lande.

14. Evangel. Kirchen in Südwesteuropa.

1 5. Evangel. Kirchen in Nordeuropa.

16. Evangel. Kirchen in Osteuropa.

17. Anglikanismus.

18. Presbyterianismus.

19. Englische Inspirationsgemeinschaften.

20. Englische Evangelisationsgemeinschaften.

2 I. Englischer Biblizismus.

22. Englischer Chiliasmus.

23. Englischer Rationalismus und Okkultismus.

24. Evangelische Landeskirchen u. nationale Kirchenbünde außerhalb Europas. 


\title{
CORPUS CONFESSIONUM DIE BEKENNTNISSE DER CHRISTENHEIT
}

\section{SAMMLUNG GRUNDLEGENDER URKUNDEN AUS ALLEN KIRCHEN DER GEGENWART}

\author{
IN VERBINDUNG MIT
}

D. ALFRED ERNEST GARVIE, Direktor des Hackney and New College in London, D. Dr. GERMANOS, Erzbischof von Thyateira, D. ARTHUR C. HEADLAM, Bischof von Gloucester, D. ADOLF KELLER, Sekretär des Schweizerischen Evangelischen Kirchenbundes, D. CHARLES S. MACFARLAND, Generalsekretür des Federal Council of the Churches of Christ in America und D. Dr. NATHAN SÖDERBLOM, Erzbischof von Upsala

HERAUSGEGEBEN VON

D. CAJUS FABRICIUS

PROFESSOR DER THEOLOGIE AN DER UNIVERSITÁT BERLIN

20. ABTEILUNG: ENGLISCHE EVANGELISATIONSGEMEINSCHAFTEN

I. BAND: DIE BISCHŐFLICHE METHODISTENKIRCHE

\author{
BERLIN UND LEIPZIG 1931
}

VERLAG VON WALTER DE GRUYTER \& CO. vormals G. J. GósChen'SChe verLagshandLung - J.GUtTENTAG, verlagsbuChHANDLUNG - GEORG REIMER - KARL J. TRÖBNER - VEIT \& COMP. 


\title{
DIE BISCHÖFLICHE METHODISTENKIRCHE IHRE KIRCHENORDNUNG UND IHRE KATECHISMEN
}

\author{
BEARBEITET VON
}

\author{
D. CAJUS FABRICIUS \\ PROFESSOR DER THEOLOGIE AN DER UNIVERSITAT BERLIN
}

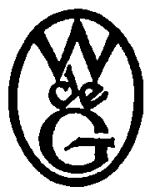

BERLIN UND LEIPZIG 1931

VERLAG VON WALTER DE GRUYTER \& CO. VORMALS G. J. GOSCHEN'SCHE VERLAGSHANDLUNG - J. GUTTENTAG, VERLAGSBUCHHANDLUNG - GEORG REIMER - KARL J. TRUBNER - VEIT \& COMP. 
Druck von Walter de Gruyter Con $_{a}$ Berlin W 10 